\title{
Can expectancies produce placebo effects for implicit learning?
}

\author{
Ben Colagiuri • Evan J. Livesey • Justin A. Harris
}

Published online: 18 November 2010

(C) Psychonomic Society, Inc. 2010

\begin{abstract}
The placebo effect is an important phenomenon whereby real changes occur in response to an otherwise inert intervention. Despite increasing research attention, it remains unclear exactly which processes are amenable to placebo effects. The current study tested whether an instructional manipulation could produce placebo effects on a nonconscious cognitive task, namely implicit learning. Four hundred and sixty-four university students completed a visual search task while smelling an odor or no odor, in alternating blocks. Unknown to them, the task contained a contingency whereby on half the trials the target's location was cued by the pattern of distractors, which was achieved by repeating some configurations of targets and distractors. Prior to the task, participants received positive, negative, or no information about the odor's possible effects on performance. Those given positive information demonstrated faster reaction times on cued trials than other participants. Those given negative information showed slower reaction times on cued trials compared with participants given no information. Further, the cuing effect appeared to be nonconscious, with participants' ability to recognize the repeated configurations equivalent to chance and no evidence that performance on a recognition test was related
\end{abstract}

B. Colagiuri · E. J. Livesey $\cdot$ J. A. Harris

School of Psychology, University of Sydney,

Sydney, Australia

B. Colagiuri $(\bowtie)$

Centre for Complementary Medicine Research,

University of Western Sydney,

Campbelltown Building 5 Locked Bag 1797 Penrith South

DCNSW 1797, Sydney, Australia

e-mail: b.colagiuri@uws.edu.au

B. Colagiuri

e-mail: bencolagiuri@yahoo.com.au to the magnitude of the cuing effect. This suggests that instructional manipulations can produce placebo effects on some nonconscious processes.

Keywords Placebo effect - Expectancy - Implicit learning · Contextual cuing

\section{Can expectancies produce placebo effects for implicit learning?}

The placebo effect is an important phenomenon whereby real psychological and/or physiological changes occur in response to an otherwise inert intervention. In the archetypical example, saline injections administered to wounded soldiers under the guise of a powerful analgesic significantly reduced the soldiers' pain (Beecher 1959; and see Colloca \& Benedetti, 2006; Kaptchuk et al., 2006 for more recent examples). While the majority of research on the placebo effect has focused on clinical settings, there is growing evidence of placebo effects in other areas, such as cognitive performance (e.g., Colagiuri \& Boakes, 2010; Fillmore, Carscadden, \& Vogel-Sprott, 1998). Although some researchers separate positive and negative effects into placebo and nocebo effects, respectively (e.g., Brody \& Brody, 2000; Shapiro \& Shapiro, 1997), this distinction leads to some logical inconsistencies (Stewart-Williams \& Podd, 2004). Therefore, we consider both positive and negative effects to constitute the placebo effect.

Traditionally, two theories have been invoked to explain the placebo effect: classical conditioning and expectancy theory. The classical conditioning account (Herrnstein, 1962; Wickramasekera, 1980) considers the placebo effect a conditioned response brought about by previous pairings of the conditioned stimulus (e.g., a syringe) and the 
unconditioned stimulus (e.g., morphine). Expectancy theory proposes that expecting an outcome is sufficient to produce that outcome in and of itself (Kirsch, 1985, 1997, 1999). For example, simply expecting pain relief leads to analgesia. While these two accounts were often treated as opposing, most researchers now agree that both classical conditioning and expectancy can contribute to a placebo effect (Brody \& Brody, 2000; Evans, 2003; StewartWilliams \& Podd, 2004), acknowledging that neither on its own can explain all placebo effects found in humans and other animals and that classical conditioning in humans is usually accompanied by conscious expectancies (Lovibond \& Shanks, 2002).

At least two integrative models of the placebo effect exist. In the first, Benedetti et al. (2003) propose that classical conditioning and expectancy can produce placebo effects on conscious physiological processes, e.g., pain, whereas only classical conditioning can produce placebo effects on nonconscious physiological processes, e.g., hormone secretion. In the second, Stewart-Williams and Podd (2004) propose that classical conditioning and expectancy can produce placebo effects on both physiological and subjective processes, but that any such expectancyinduced placebo effects are always consciously mediated, whereas placebo effects resulting from classical conditioning can be either consciously or nonconsciously mediated.

Support for both of these models comes from two experiments conducted by Benedetti et al. (2003). In the first, healthy participants received pre-training with analgesic injections while undergoing pain sensitivity measurements over 2 days, thus allowing for an association between receiving an injection and pain relief. On a subsequent day, these participants were given a placebo injection containing saline only. One group was told that the injection contained the same analgesic they had received on the previous 2 days, while a second group was told that it contained a hyperalgesic agent that would increase their pain. The first group showed increased pain tolerance after the placebo injection, while the second showed decreased pain tolerance, even though they had received the same pre-training. Thus, the placebo-induced changes in pain tolerance (considered a conscious process) were mainly affected by expectancy. The second experiment applied the same design, but used sumatriptan, which increases growth hormone levels (GHL), rather than an analgesic. Here the pre-training allowed for the formation of an association between the injection and increased GHL. This second experiment revealed increases in GHL in response to the placebo injection regardless of what participants were told, indicating no effect of expectancy (but an effect of conditioning). Importantly, the participants were found to be unaware of changes in their GHL, suggesting that this conditioned placebo effect was nonconscious. Note that neither experiment included pre-training control groups administered with placebo only.

Benedetti et al. (2003) and Stewart-Williams and Podd's (2004) models raise interesting questions about which types of processes are amenable to placebo effects. One such possibility that remains untested is whether or not expectancy can produce placebo effects for nonconscious cognitive processes, such as implicit learning. The current study investigated this possibility by manipulating information given to participants prior to undertaking a visual search task that involved an implicit learning component, namely contextual cuing (Chun \& Jiang, 1998, 2003). On each trial of this task participants search for the letter $\mathrm{T}$ that has been rotated by 90 degrees either clockwise or anticlockwise. This target is hidden in a background (context) consisting of a number of letter Ls (distractors) that are rotated by either $0,90,180$, or 270 degrees, and scattered throughout the visual field in which the rotated $\mathrm{T}$ is located. The participants' task is to locate the T and then respond by indicating whether it is rotated clockwise or anticlockwise. Some configurations are repeated, and thus for these configurations the arrangement of the distractors serves as a cue for the location of the target. Contextual cuing occurs when reaction times on cued trials are faster than when the target's location is not cued.

An important feature of contextual cuing is its persistence even in the absence of conscious recognition of the repeated configurations. Many contextual cuing studies have used post-experiment tests to gauge explicit knowledge of the relationship between target position and distractor configuration, typically using one of two measures: recognition of the repeated contexts indexed by "old" vs. "new" judgments of repeated and novel configurations (Chun \& Jiang, 1998) or generation tasks where the participant must judge where the target should be located within a given configuration of distractors (Chun \& Jiang, 2003). These two measures have yielded similar results, with many studies either failing to find evidence of better recognition for repeated configurations (Chun \& Jiang, 1998; Chun \& Phelps, 2001; Howard, Dennis, Howard, Yankovich, \& Vaidya, 2004; Manns \& Squire, 2001; Rausei, Makovski, \& Jiang, 2007) or finding generation performance equivalent to chance (Chun \& Jiang, 2003; Jiménez \& Vázquez, 2010), despite the presence of robust contextual cuing effects. In some studies where these posttest measures have detected some degree of explicit knowledge (performance above chance), contextual cuing still appears to be unaffected by the participant's level of awareness, and cuing effects have been observed reliably in subgroups of participants who show no explicit recognition (Preston \& Gabrieli, 2008; Vaidya, Huger, Howard, \& Howard, 2007). Perhaps most compelling of all, even experiments that explicitly draw participants' attention to 
the possibility of repeated configurations, either through instruction (Chun \& Jiang, 2003) or concurrent explicit testing (Smyth \& Shanks, 2008), still yield evidence of contextual cuing among participants who show no evidence of explicit knowledge of the repeated configuration. Consequently, contextual cuing is widely thought to arise automatically as a result of implicit learning.

In the present experiment, participants' expectancies about their performance on the search task were manipulated by giving positive, negative, or no information about the possible effects of an odor. They were not, however, told that the visual search task involved contingencies between the configuration of distractors and the target's location. If expectancies can affect nonconscious cognitive processes, then the suggestion of improvement or impairment should produce placebo effects on both overall performance on the visual search task and implicit learning. If, on the other hand, expectancies cannot affect nonconscious cognitive processes, then placebo effects should be observed for overall performance on the visual search task, but not for the implicit learning component if participants are found to be unaware of the relevant associations.

\section{Method}

\section{Participants}

Four hundred and sixty-four students enrolled in introductory psychology at the University of Sydney (17-56 years; $66 \%$ female) participated as part of their tutorial course. Participants were excluded if they had heard about the experiment before their tutorial $(n=10)$ or if their overall accuracy on the visual search task was less than $95 \%(n=29)$.

\section{Design}

This study employed a $3 \times(2) \times(2)$ design. The threelevel between-subjects factor was the information participants received about the possible effect of the odor on their performance: positive, negative, or no information. The first within-subjects factor was the presence or absence of an odor, with participants performing the visual search task smelling the odor or no odor on alternate blocks of trials, counterbalanced across participants. The second within-subjects factor was contextual cuing. On half of the trials in the visual search task, the configuration of distractors cued the location of the target, whereas on the other half the configuration of distractors was random and provided no information about the target location. The dependent variable was reaction time on the visual search task.

\section{Materials}

Odor Approximately $1 \mathrm{ml}$ of a 'bubblegum' odor (Quest International) was placed on a cotton pad in a specimen container. A similar but empty specimen container was used for the no-odor blocks.

Visual search task The visual search task required participants to find and report the orientation of a $90^{\circ}$ rotated letter $\mathrm{T}$ (the target), located among $15 \mathrm{~L}$-shaped distractors rotated $0,90,180$, or 270 degrees. Each target and distractor subtended a visual angle of $0.8^{\circ}$ at approximately $57-\mathrm{cm}$ viewing distance. On every trial, four items appeared in each quadrant of a square area $\left(15^{\circ}\right.$ width, shaded light gray) in the center of the computer screen such that the target and distractors were distributed evenly but in a seemingly unsystematic fashion. All distractor and target items were black. Participants were required to press one key if the target was rotated to the left and another if it was rotated to the right. Participants were told to respond as fast as possible without sacrificing accuracy. The program randomly generated eight configurations of target and distractors that were repeated throughout the experiment and comprised the cued trials. Four of these configurations (each with the target appearing in a different quadrant) were used for the odor trials and four for the no-odor trials. Throughout the experiment, half of the trials were cued, with the same fixed configuration of distractors predicting where the target would be located (but not its orientation). The other half were uncued trials, in which the target would occur an equal number of times in one of four locations, but the distractors were randomly distributed on each presentation. Contextual cuing occurs when performance is faster on cued trials than uncued trials.

\section{Procedure}

Participants were told that the study examined whether an odor could affect cognitive performance. They were given a container marked $\mathrm{O}+$, which contained the bubblegum odor, and an empty container marked NO. They were then allocated by tutorial group to one of the three information conditions (positive, negative, or no information), such that all participants within a tutorial received the same information. The positive information group was told that the odor may improve their cognitive performance by focusing attention the negative information group was told that the odor may impair their cognitive performance by distracting attention, and the no information group received no information about the odor's possible effects. Next, participants completed 10 blocks of the visual search task, each containing 32 trials, and were instructed via computer prompts to hold the $\mathrm{O}+$ 
container just below their nose with their non-dominant hand on half the blocks and to hold the NO container in a similar fashion on the remaining blocks. This meant that the four cued trials allocated to odor and the remaining four allocated to no odor were each presented a total of 20 times. Finally, participants completed an explicit recognition test. As discussed, the most commonly used measure of explicit knowledge in contextual cuing is a post-experiment recognition test. We employed this procedure but used a twoalternative forced choice (2AFC) recognition task rather than an "old" vs. "new" judgment. Participants were presented with one repeated configuration and one configuration in which the target location was shifted to a position in the symmetrically opposite quadrant of the screen. One distractor in this quadrant was also shifted to a new position within the quadrant previously occupied by the target. Hence, the resulting foil was a configuration in which the distractortarget relationship was violated. Participants were told that one of the configurations (and one only) had been presented repeatedly during the experiment, and they were asked to identify the repeated configuration. The $2 \mathrm{AFC}$ procedure was adopted to increase the sensitivity of the test, in particular avoiding the concern that participants are sometimes biased towards reporting that most or all configurations are new. Both explicit recognition of the repeated configuration and explicit knowledge of the distractor-target relationships could aid performance in this test. Each of the eight repeated configurations was tested twice in this fashion (162AFC trials in total).

\section{Statistical analysis}

Contrast analysis controlling for order of administration tested whether reaction times differed for odor versus no odor and/or the expectancy manipulation, and whether these two factors interacted. For the expectancy manipulation, this involved two mutually orthogonal contrasts comparing positive information with other information, and negative versus no information. Contrast analysis tested whether reaction times differed on cued versus uncued trials overall, controlling for order of administration. Difference scores between reaction times on cued and uncued trials were then calculated, and further contrast analyses tested differences in cuing across odor presentation, instruction group, and their interaction, controlling for order of administration. Given that participants were excluded if they achieved less than $95 \%$ accuracy on the visual search task, we only analyzed errors to ensure that any findings for reaction time were not caused by a speedaccuracy trade-off. This was achieved by repeating the above analysis for errors.

To investigate whether contextual cuing occurred in the absence of awareness, we conducted a single sample $t$-test to determine whether contextual cuing occurred in participants who performed at or below chance on the recognition test. We also tested whether there were differences in the magnitude of the contextual cuing effect for participants performing at or below chance $(8 / 16$ or less correct) on the recognition test compared with those performing at or above $68 \%(11 / 16$ correct) or at or above $80 \%$ (13/16 correct). Finally, contrast analysis compared recognition of the repeated configurations across information groups. Results were considered statistically significant when $p<.05$.

\section{Results}

Figure 1 shows overall reaction times for odor and no-odor trials across groups. Overall, participants demonstrated faster search times with the odor present than in its absence, $\mathrm{F}(1,421)=140, p<.001, \eta_{\mathrm{p}}^{2}=.25$. Participants given positive information demonstrated reaction times $26.9 \mathrm{~ms}$ faster than those given negative or no information, on

\section{A. Reaction Time Overall}

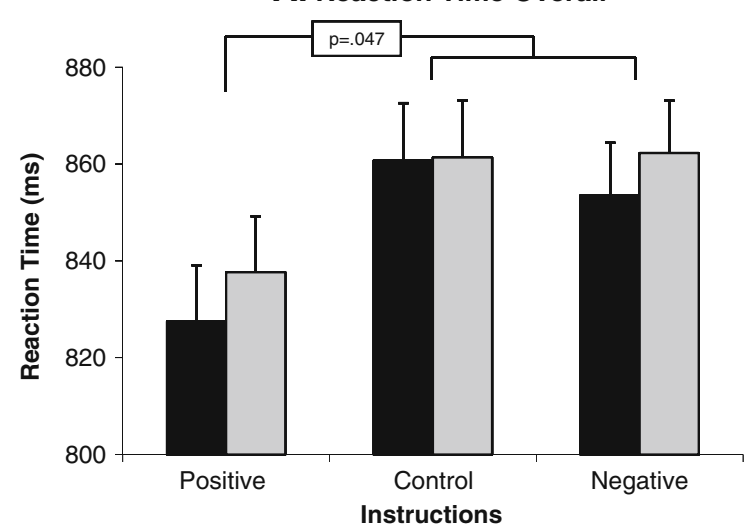

\section{B. Errors Overall}

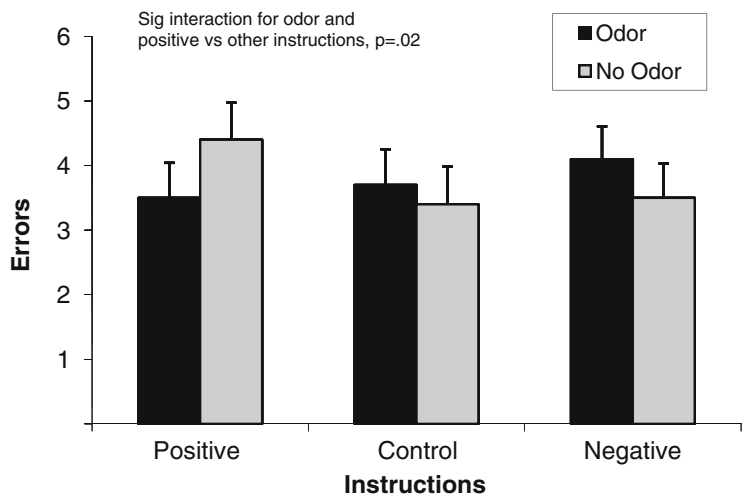

Fig. 1 Mean (+SE) overall reaction time on the visual search task on odor and no-odor trials across the three information conditions 
average, $\mathrm{F}(1,421)=3.98, p=.047, \eta_{\mathrm{p}}{ }^{2}=.01$. There were no significant differences in reaction times between the negative and no information groups, nor any significant interactions between odor and information, all $\mathrm{F}<1$. As for the overall error rates shown in Figure 1b, no main effects were found for odor or information, all $\mathrm{Fs}<1$. A significant interaction between odor and positive versus other information indicated that participants given positive information made fewer errors on odor trials, while the opposite occurred for negative or no information, $\mathrm{F}(1,421)=5.54, p=.02$. Thus, there did not appear to be any speed-accuracy trade-off for overall performance.

Figure 2 shows mean reaction time for cued versus uncued trials across blocks separately for each group. There was a significant overall contextual cuing effect with participants identifying the target $22.9 \mathrm{~ms}$ faster on cued trials than uncued trials, $\mathrm{F}(1,421)=13.2, p<.001, \eta_{\mathrm{p}}{ }^{2}=$ .03 . Figure $3 \mathrm{a}$ shows the magnitude of the cuing effect measured as the difference between mean reaction times for cued and uncued trials averaged across blocks. Contrast analysis revealed that participants who received positive information demonstrated an average cuing effect $16.2 \mathrm{~ms}$ greater than negative or no information, $\mathrm{F}(1,421)=5.21$, $p=.02, \eta_{\mathrm{p}}{ }^{2}=.01$. Participants who received negative information showed a cuing effect $18.0 \mathrm{~ms}$ smaller than those who received no information $\mathrm{F}(1,421)=4.92, p=.03$, $\eta_{\mathrm{p}}{ }^{2}=.01$. There were no significant differences in cuing on odor versus no-odor trials, $\mathrm{F}(1,421)=2.42, p=.12$, nor were there any significant interactions, both $\mathrm{F}<1$.

Figure $3 \mathrm{~b}$ shows contextual cuing for errors across odor and information. The cuing was stronger, i.e., fewer errors on cued than uncued trials, for those given positive information compared with negative or no information, $\mathrm{F}(1,421)=5.52, p=.02$. The main effect of odor on cuing of errors was not statistically significant, $\mathrm{F}(1,421)=$ $3.19, p=.08$. As with overall errors, there was a significant interaction between information and odor suggesting that the larger cuing effect for positive information compared to other information was more marked on odor than no-odor trials, $\mathrm{F}(1,421)=5.13, p=.02$. Therefore, as with overall performance, there was no evidence of a speed-accuracy trade-off for contextual cuing. The results for errors were identical when participants were included who performed below $95 \%$ accuracy.

In the explicit recognition test, mean recognition of repeated configurations was $50.2 \%$, which is equivalent to chance. Further, there were no significant differences in recognition across information groups, $\mathrm{F}<1$. Of the 423 participants included in the study, 264 scored $8 / 16$ or lower (expected $\mathrm{n}$ from a binomial distribution $=276$ ). Looking solely at this sample of participants who failed to score better than chance, there was still a highly significant cuing effect of $19.9 \mathrm{~ms}, \mathrm{t}(263)=4.52, p<.001$. Curiously, although fewer participants scored above 50\% correct $(>8 / 16)$ than expected by chance (159 vs. 170 expected from binomial distribution), a disproportionate number of these participants achieved relatively high scores. Sixty-two participants (cf. 44 expected by chance) scored 11 or higher, of which 11 (cf. 4-5 expected by chance) scored 13 or higher. However, when compared to the 264 participants who scored at or below chance, the cuing effect was not statistically greater for either the 62 participants scoring 11
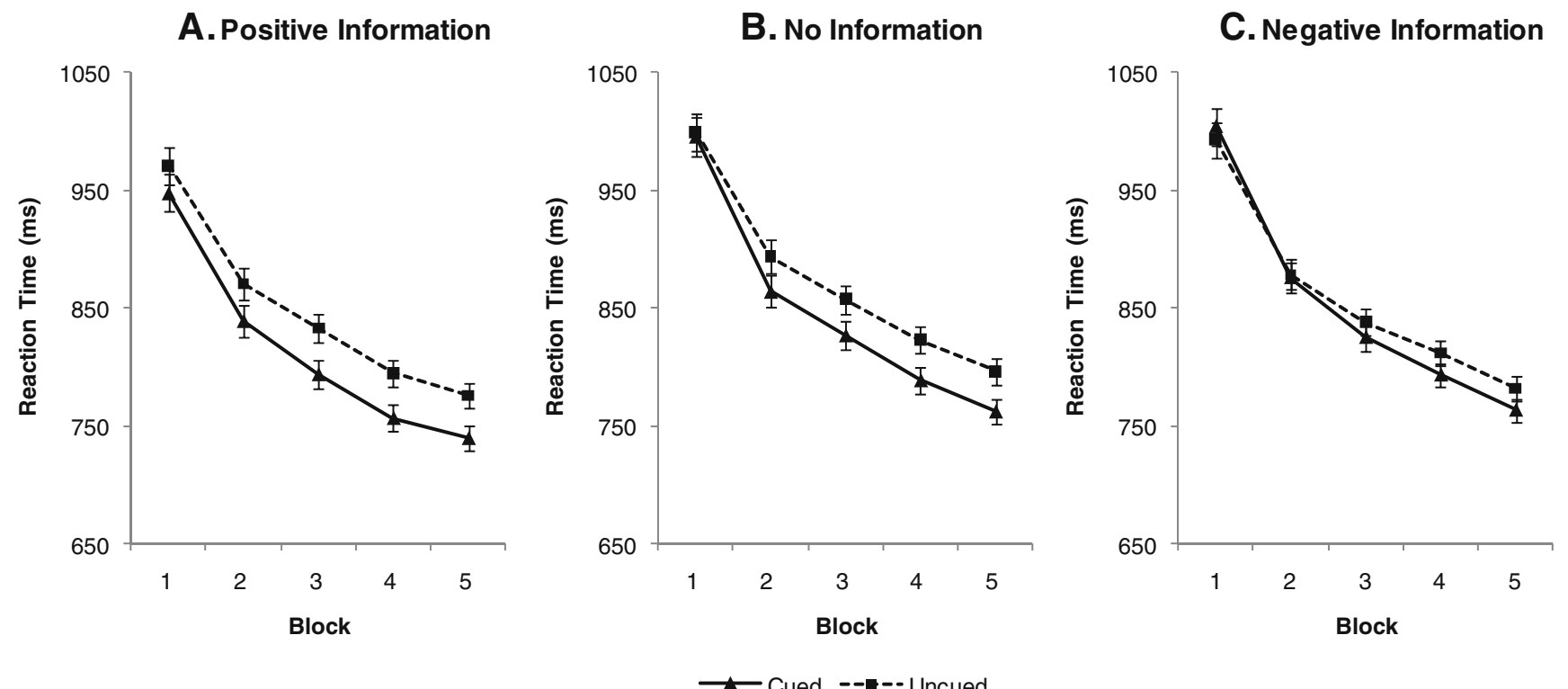

Fig. 2 Mean $( \pm \mathrm{SE})$ reaction time for cued versus uncued trials across blocks and displayed separately for those receiving positive information (a), no information (b), and negative information (c) 
A. Contextual Cuing of Reaction Time

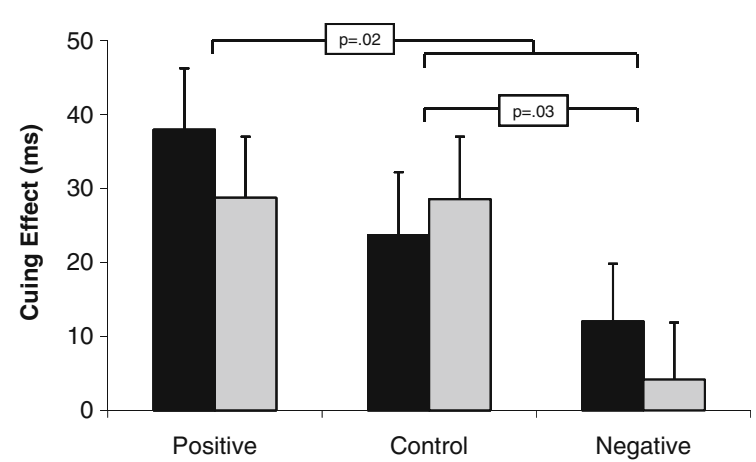

B. Contextual Cuing of Errors

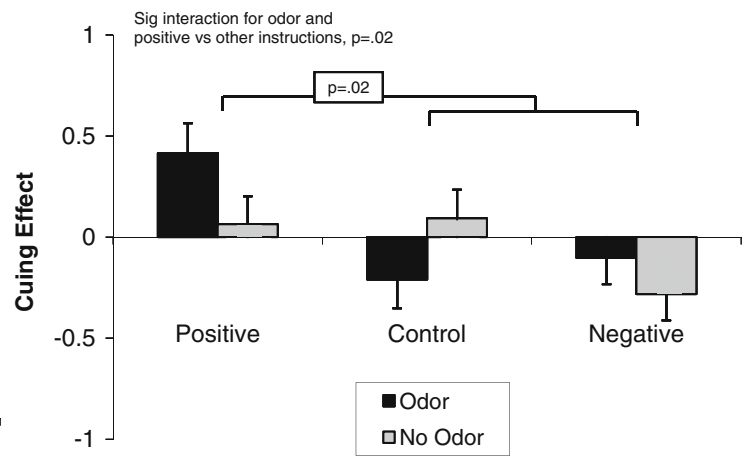

Fig. 3 Mean (+SE) cuing effect for reaction time (a) and errors (b) on the visual search task on odor and no-odor trials across the three information conditions. In both cases higher scores indicate a larger cuing effect, i.e. better implicit learning

or higher or the 12 participants who scored 13 or higher, both $\mathrm{t}<1$.

\section{Discussion}

The most interesting result of this study was that information provided to participants about possible effects of an odor influenced implicit learning. The pattern of results from the recognition tests were consistent with the contextual cuing literature (e.g., Chun \& Jiang, 1998; Chun \& Phelps, 2001; Howard et al., 2004; Manns \& Squire, 2001; Rausei et al., 2007) and the notion that cuing is driven largely by implicit learning and memory processes. Positive information increased the magnitude of contextual cuing of reaction times compared with negative or no information, while negative information led to a smaller cuing effect than no information. Further, the analysis of errors indicated that this effect could not be explained by a speed-accuracy trade-off. These findings suggest that expectancies induced via an instructional manipulation can influence nonconscious cognitive processes.

Another interesting observation was that the placebo effects observed in the two experimental groups did not perfectly mirror the information with which they were provided. Participants in these groups were informed that their performance may increase or decrease for the odor compared with no odor. However, for reaction times there were no significant interactions between odor and information. Instead, participants given positive information responded faster overall and demonstrated stronger cuing than other information groups, and those given negative information demonstrated weaker cuing than no information, regardless of the presence or absence of the odor. This suggests that the information may have produced general expectancies for improvement or decrement rather than expectancies about specific differences between the odor and no odor. There are two ways in which this may have occurred. First, participants may not have fully attended to or remembered all of the information provided and, if so, may have only registered that their performance might be enhanced or impaired, without understanding the suggestion that this would be specific to trials with the odor. Alternatively, even if participants fully comprehended the information, they may have expected the odor to have effects that endured beyond the exact moment that they sniffed it. A general placebo effect that is independent of the presence of the odor may seem inconsistent with conventional notions of the placebo effect that necessarily entail the administration of a treatment. However, more recent conceptions of the placebo effect suggest that it does not require the administration of a placebo per se (e.g., Di Blasi, Harkness, Ernst, Georgiou, \& Kleijnen, 2001; Moerman \& Jonas, 2002). As an example of such a placebo effect, naval cadets who received bogus instruction that they were more likely to suffer seasickness did actually experience higher levels of seasickness compared with naval cadets who received no such instruction (Eden \& Zuk, 1995).

The present study shows that contextual cuing could provide a useful method for testing placebo effects and their characteristics. Most evidence for placebo effects is based on subjective outcomes. While this may be unavoidable given that many symptoms of health and illness cannot be measured objectively, it does mean that participant bias may confound results, particularly bias produced by the demand characteristics of the study (Kienle \& Kiene, 1997). Further, even objective outcomes, such as cognitive performance, may be influenced by demand characteristics, especially because most placebo manipulations involve a clear description of what the participants should expect. Given the nonconscious nature of implicit learning, it is highly unlikely that it could be affected by demand characteristics and may, therefore, provide more robust evidence for the placebo effect. Of course, even when implicit learning is involved it is possible 
that other forms of bias, such as motivation, could influence outcomes. Nonetheless, implicit learning should reduce the opportunities for bias compared with tasks involving only conscious processes.

Acknowledgment We would like to thank Prof. Robert Boakes for his useful comments on an earlier draft. We have no conflicts of interest in producing this report.

\section{References}

Beecher, H. K. (1959). The powerful placebo. Journal of the American Medical Association, 159, 1602-1606.

Benedetti, F., Pollo, A., Lopiano, L., Lanotte, M., Vighetti, S., \& Rainero, I. (2003). Conscious expectation and unconscious conditioning in analgesic, motor, and hormonal placebo/nocebo responses. The Journal of Neuroscience, 23, 4315-4323.

Brody, H., \& Brody, D. (2000). Three perspectives on the placebo response: Expectancy, conditioning, and meaning. Advances in Mind-Body Medicine, 16, 216-232.

Chun, M. M., \& Jiang, Y. (1998). Contextual cuing: Implit learning and memory of visual context guides spatial attention. Cognitive Psychology, 36, 28-71.

Chun, M. M., \& Jiang, Y. (2003). Implicit, long-term spatial contextual memory. Journal of Experimental Psychology. Learning, Memory, and Cognition, 29, 224-234.

Chun, M. M., \& Phelps, E. A. (2001). Memory deficits for implicit contextual information in amnesic subjects with hippocampal damage. Nature Neuroscience, 2, 844-847.

Colagiuri, B., \& Boakes, R. A. (2010). Perceived treatment, feedback, and placebo effects in double-blind RCTs: An experimental analysis. Psychopharmacology, 208, 433-441.

Colloca, L., \& Benedetti, F. (2006). How prior experience shapes placebo analgesia. Pain, 124, 126-133.

Di Blasi, Z., Harkness, E., Ernst, E., Georgiou, A., \& Kleijnen, J. (2001). Influence of context effects on health outcomes: A systematic review. [Meta-Analysis

Eden, D., \& Zuk, Y. (1995). Seasickness as a self-fulfilling prophecy: Raising self-efficacy to boost performance at sea. The Journal of Applied Psychology, 80, 628-635.

Evans, D. (2003). Placebo: The Belief Effect. London: Harper Collins.

Fillmore, M. T., Carscadden, J. L., \& Vogel-Sprott, M. (1998). Alcohol, cognitive impairment and expectancies. Journal of Studies on Alcohol, 59, 174-179.

Herrnstein, R. (1962). Placebo effect in the rat. Science, 138, 677678.
Howard, J. H., Dennis, N. A., Howard, D. V., Yankovich, H., \& Vaidya, C. J. (2004). Implicit spatial contextual learning in healthy aging. Neuropsychology, 18, 124-134.

Jiménez, L., \& Vázquez, G. A. (2010). Implicit sequence learning and contextual cuing do not compete for central cognitive resources. Journal of Experimental Psychology: Human Perception and Performance. Epub ahead of print, 23rd August 2010.

Kaptchuk, T. J., Stason, W. B., Davis, R. B., Legedza, A. R. T., Schnyer, R. N., Kerr, C. E., et al. (2006). Sham device v inert pill: Randomised controlled trial of two placebo treatments. British Medical Journal, 332, 391-397.

Kienle, G. S., \& Kiene, H. (1997). The powerful placebo effect: Fact of fiction? Journal of Clinical Epidemiology, 50, 1311-1318.

Kirsch, I. (1985). Response expectancy as a determinant of experience and behaviour. The American Psychologist, 40, 1189-1202.

Kirsch, I. (1997). Specifying nonspecifics: Physiological mechanisms of placebo effects. In A. Harrington (Ed.), The Placebo Effect: An Interdisciplinary Exploration (pp. 166-186). Massachusetts: Harvard University Press.

Kirsch, I. (1999). How Expectancies Shape Experience. Washington: American Psychological Association.

Lovibond, P. F., \& Shanks, D. R. (2002). The role of awareness in pavlovian conditioning: Empricial evidence and theoretical implications. Journal of Experimental Psychology, 28, 3-26.

Manns, J. R., \& Squire, L. R. (2001). Perceptual learning, awareness, and the hippocampus. Hippocampus, 11, 776-782

Moerman, D. E. P., \& Jonas, W. B. M. D. (2002). Deconstructing the Placebo Effect and Finding the Meaning Response. Annals of Internal Medicine, 136, 471-476.

Preston, A. R., \& Gabrieli, J. D. E. (2008). Dissociation between explicit memory and configural memory in the human medial temporal lobe. Cerebral Cortex, 18, 2192-2207.

Rausei, V., Makovski, T., \& Jiang, Y. V. (2007). Attention dependency in implicit learning of repeated search context. Quarterly Journal of Experimental Psychology, 60, 1321-1328.

Research Support, Non-U.S. Gov't

Research Support, U.S. Gov't, P.H.S.]. Lancet, 357, 757-762.

Shapiro, A. K., \& Shapiro, E. (1997). The Powerful Placebo: From Ancient Priest to Modern Physician. Baltimore: The John Hopkins University Press.

Smyth, A. C., \& Shanks, D. R. (2008). Awareness in contextual cuing with extended and concurrent explicit tests. Memory \& Cognition, 36, 403-415.

Stewart-Williams, S., \& Podd, J. (2004). The placebo effect: Dissolving the expectancy versus conditioning debate. Psychological Bulletin, 130, 324-340.

Vaidya, C. J., Huger, M., Howard, D. V., \& Howard, J. H. (2007). Developmental differences in implicit learning of spatial context. Neuropsychology, 21, 497-506.

Wickramasekera, I. (1980). A conditioned response model of the placebo effect: Predictions from the model. Biofeedback and Self Regulation, 5, 5-18. 